\title{
HOLLOW CORE FIBERS FOR OPTICAL AMPLIFICATION
}

\author{
Walter Belardi $^{1 *}$, Pier J. Sazio ${ }^{2}$, Laurent Bigot ${ }^{1}$ \\ ${ }^{1}$ Université de Lille, CNRS, UMR 8523 -PhLAM - Physique des Lasers, Atomes et Molécules, F-59000 Lille, France \\ ${ }^{2}$ Optoelectronics Research Centre, University of Southampton, Highfield, Southampton, Hampshire. SO17 1BJ, UK \\ *Corresponding author: walter.belardi@univ-lille.fr
}

Received XX Month XXXX; revised XX Month, XXXX; accepted XX Month XXXX; posted XX Month XXXX (Doc. ID XXXXX); published XX Month XXXX

\begin{abstract}
Hollow core optical fibers are normally passive light transport components. In contrast, within this study, we numerically investigate the possibility of using them as optical amplifiers, through the adoption of a novel fiber structure. We show that optical amplification can be achieved in hollow core fibers where the cladding region is partially doped and composed of both resonant and antiresonant elements. A balance between loss and glass/optical mode overlap is obtained, which allows efficient amplification over a limited spectral bandwidth. We discuss the case of a Thulium-doped optical amplifier based on this novel technological approach. (C) 2019 Optical Society of America
\end{abstract}

OCIS codes: (060.2280) Fiber design and fabrication; (060.2400) Fiber properties; (060.4005) Microstructured fibers

http://dx.doi.org/10.1364/OL.99.099999

Hollow Core Optical Fibers (HCs) are a specific type of specialty optical fiber which allows efficient optical propagation in vacuum (or air), thus attracting the interest of a large scientific community [1]. In particular, during the last few years, this interest has been stimulated by the development of simplified $\mathrm{HC}$ structures with only one ring of detached tubes surrounding a central air core [2]. In this specific type of HCs, light propagation is assured by the fact that the optical thickness of the silica layer surrounding the air core satisfies an anti-resonance condition [3-5]. This specific type of HCs is also referred to as HC Anti-Resonant Fiber (ARF) and its applications range from Gas Photonics [6], Mid-infrared optics [7], Optical Communications [8] to High Power optical beam delivery [9] and others [10-12]. All of these applications exploit the possibility in an ARF of having a minimal interaction between the air-guided optical mode and the glass elements in the surrounding cladding area. Most often the overlap between the fundamentallike optical mode and the glass is typically less than $0.01 \%$ [13-15, 6].

Despite the majority of uses for ARFs as passive photon transport optical components, several investigations have concerned the development of gas-based ARF lasers, both optically or electrically pumped [16, 17, 2]. Filling the core of an ARF with a gas phase compound (e.g. acetylene, $\mathrm{C}_{2} \mathrm{H}_{2}$ ) can be an efficient method for obtaining optical amplification by exploiting nonlinear phenomena (such as Stimulated Raman Scattering), or even population inversion $[18,2]$. Similarly, an additional layer of material can be deposited within a HC fiber for its functionalisation [19-21]. In contrast, obtaining optical amplification in an unfilled HC (or filled with air), would require to exploit the glass properties in the cladding area and thus would imply a reasonable level of overlap between the core-guided optical mode and the glass.

The first investigations into the concept of a hollow core optical fiber amplifier (HC-OFA) were made in [22] (fabrication and implementation) and [23] (theory). In that work, the potential advantages of an Ytterbium doped HC-OFA were related to the ability of a HC fiber to handle very high optical power without being compromised by nonlinear effects or glass breakdown. Today, the rapid development of HC fiber technology is hitting the field of optical communications [8] and can be interesting to study the characteristics of a Thulium (Tm) doped HC-OFA, operating around a wavelength of $2 \mu \mathrm{m}$ [24]. Indeed, HCs fiber attenuation (a critical parameter in the development of optical communication systems) is mainly governed, at short wavelengths, by light scattering, and largely decreases at longer wavelengths [25].

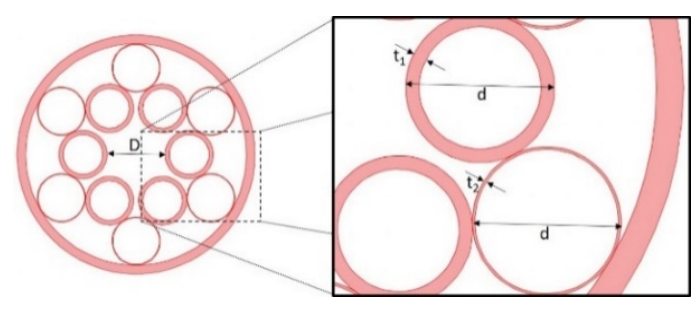

Fig. 1. HC fiber with resonant and anti-resonant elements.

Such Tm-doped HC-OFA may be used within a HC based optical communication link in order to reduce the optical impedance in the connection between passive and active components, as a way for reducing the mode mismatch between them and, also, in order to reduce the overall optical latency of the full optical system.

Here we propose, for the first time, an HC-OFA based on the use of a novel optical structure design shown in Fig. 1. This ARF comprises two rings of cladding tubes surrounding a central air core with diameter $D=50 \mu \mathrm{m}$. The internal ring is composed of 6 
Tm-doped silica tubes with external diameter $d=41.6 \mu \mathrm{m}$ and thickness $t_{1}=3.76 \mu \mathrm{m}$, while the outer ring is composed of 6 undoped silica tubes, disposed as shown in Fig. 1, with external diameter $d$ and thickness $t_{2}=0.7 \mu \mathrm{m}$. The choice of this particular structure is given by the need of increasing the overlap of the fundamental-like guided mode on the Tm-doped glass elements, with minor impacts on the overall light attenuation. Indeed, typical ARFs have a fraction of Power On Glass (POG) lower than $0.01 \%$ $[7,13,14]$. With such a low overlap, it wouldn't be viable to exploit optical pumping of the doped cladding glass material and to amplify light at the signal wavelength. In contrast, within this novel design, the inner cladding tubes thickness $t_{1}$ is chosen to be in resonance with the pump and signal wavelengths [5], so that the POG of the fundamental mode on the inner cladding tubes can be increased. On the other hand, the thickness of the outer cladding tubes $t_{2}$ is chosen to be in anti-resonance with both the pump and signal wavelengths [5], so that the overall confinement of the fundamental-like mode within the optical fiber can be preserved with relatively low attenuations at both wavelengths. The novel design results then in a new type of ARF in which both resonant and anti-resonant elements are included within the fiber structure. As in previous works $[7,13,14]$ we use Comsol Multiphysics to perform our numerical simulations. Sellmeier equations are adopted for the refractive index calculation of all the glass elements, neglecting the impact of Tm doping on the refractive index of the internal tubes. The results are shown in Fig. 2, at the pump (A) and signal wavelength (B).
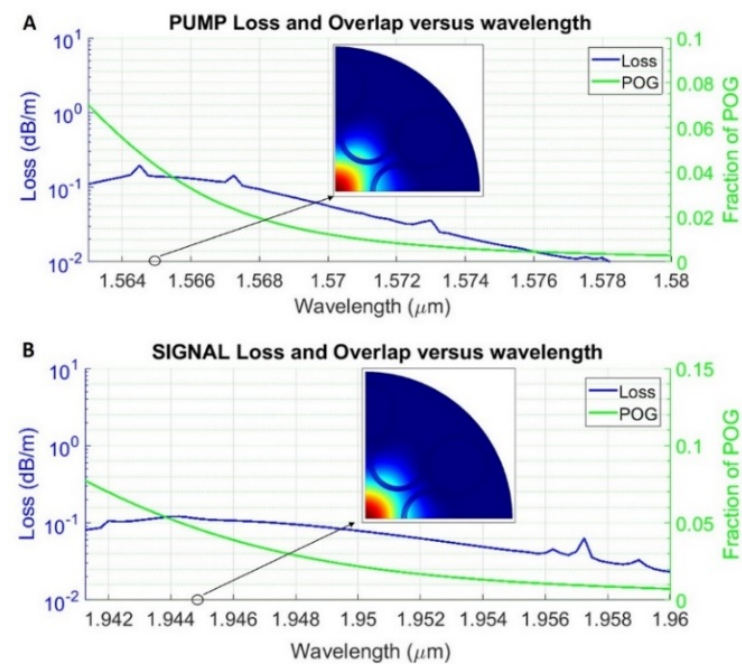

Fig. 2. Loss (blue lines, left-hand axis) and fraction of POG (green lines, right-hand axis) at pump (A) and signal (B) wavelengths.

The analyzed wavelength range for the pump (1.563-1.58 $\mu \mathrm{m})$ and for the signal (1.941-1.96 $\mu \mathrm{m}$ ) are within the absorption and emission bands of $\mathrm{Tm}^{3+}$ ions embedded in a silica glass. In both Fig. 2A (top) and Fig. 2B (bottom) it is shown the dependence of the loss (in blue, left-hand scale) and the fraction of POG (in green, right-hand scale) on the wavelength of operation. We have verified that, within the analyzed wavelength range, the lowest loss mode is localized in the air core and is the fundamental-like optical mode. In particular, the optical modes corresponding to the pump wavelength of $1.565 \mu \mathrm{m}$ and to the signal wavelength of $1.945 \mu \mathrm{m}$ are shown in the insets of Fig. 2. As we can see, for the pump (Fig. 2A), the proposed structure allows us to obtain a maximum overlap of more than $7 \%$ in combination with an attenuation lower than $0.11 \mathrm{~dB} / \mathrm{m}$, in the region around $1.563 \mu \mathrm{m}$. Note that a fraction of POG between 0.01 and 0.07 is obtained in combination with losses for the fundamental air core mode lower than 0.2 $\mathrm{dB} / \mathrm{m}$, over a spectral range about $8 \mathrm{~nm}$ wide. As for the signal (Fig. 2B), a maximum overlap of $7.8 \%$ is obtained in combination with a loss of $0.08 \mathrm{~dB} / \mathrm{m}$, in the region around $1.941 \mu \mathrm{m}$. A fraction of POG comprised between 0.01 and 0.078 , together with losses of the fundamental air mode lower than $0.11 \mathrm{~dB} / \mathrm{m}$, is reached over $16 \mathrm{~nm}$ spectral range. Note that, given the level of leakage losses and overlap achieved, it is possible to neglect completely the impact of both surface scattering and material attenuation on the total loss of the fiber, at both the pump and signal wavelengths.
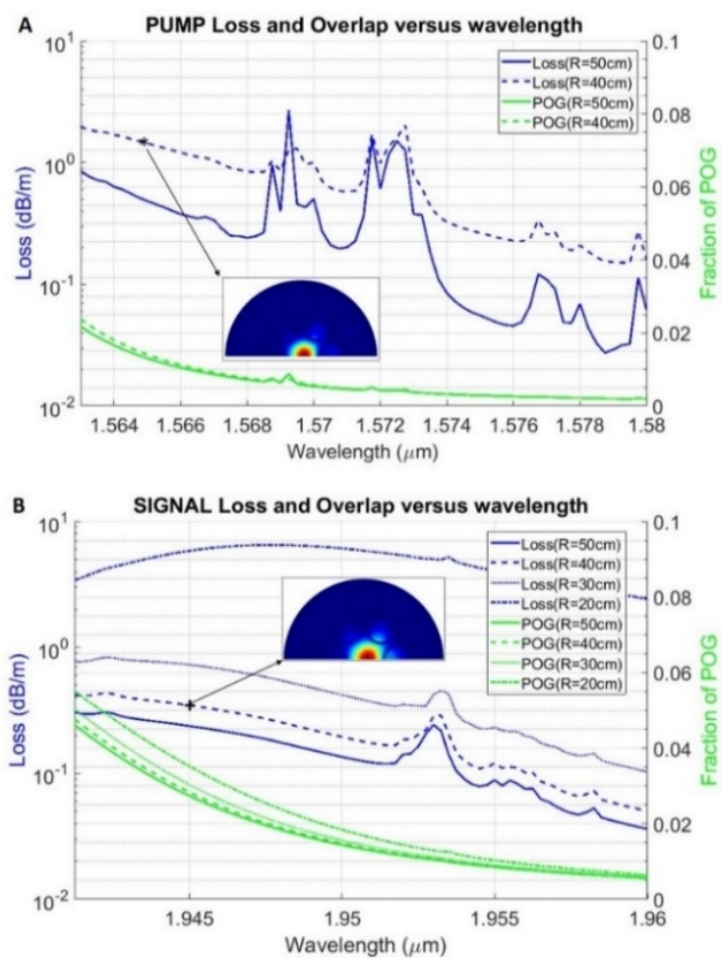

Fig. 3. Evolution of loss (blue lines, left-hand axis) and fraction of POG (green lines, right hand axis) for the pump (A) and signal (B) at different bend radii $R$.

Similarly, to previous works [7], we now analyze what happens when the proposed structure is bent with a bending radius $R$. Fig. 3 shows, like Fig. 2, the wavelength dependence of the losses (in blue) and of the fraction of POG (in green) for both the pump(A) and signal (B) wavelengths. The upper plot (Fig. 3A) represents the behaviour of the fiber at the pump wavelength, for the bending radius $R$ of $50 \mathrm{~cm}$ (full line) and $40 \mathrm{~cm}$ (dotted line). The bottom plot (Fig.3B) shows the behaviour of the ARF at the signal wavelength, for the bending radius $R$ of $50 \mathrm{~cm}$ (full lines), $40 \mathrm{~cm}$ (dashed lines), $30 \mathrm{~cm}$ (dotted lines) and $20 \mathrm{~cm}$ (dashed/dotted lines). In the insets of Fig. 3 are shown the modes at the pump wavelength of $1.565 \mu \mathrm{m}$ (up) and at the signal wavelength of 1.945 $\mu \mathrm{m}$ (down) when the bending radius is $40 \mathrm{~cm}$. As we can see, at the 
pump wavelengths, when the bending radius decreases from 50 to $40 \mathrm{~cm}$, while the level of overlap (POG) is similar, the losses of the ARF strongly increase. If we consider a POG above $1 \%$, only the ARF with $R=40 \mathrm{~cm}$ can allow a loss below $1 \mathrm{~dB} / \mathrm{m}$ over a spectral range of about $4 \mathrm{~nm}$. In contrast to the pump wavelengths, the behaviour of the ARF at the signal wavelengths has much greater bending resistance. This is not a surprise because ARFs are known to be much more bending resistant at longer wavelengths [7]. Indeed, even with a bending radius $R$ of $30 \mathrm{~cm}$ (dotted lines) we can see that losses lower than $1 \mathrm{~dB} / \mathrm{m}$ are obtained in combination with a POG between $1 \%$ and $6.2 \%$ over a bandwidth of $14 \mathrm{~nm}$.

It is interesting to see, in Fig. 4, which other modes, besides Mode 0 (similar to a classical LP 01 mode), are supported by the fiber structure of Fig. 1, at the signal wavelength of $1.945 \mu \mathrm{m}$.

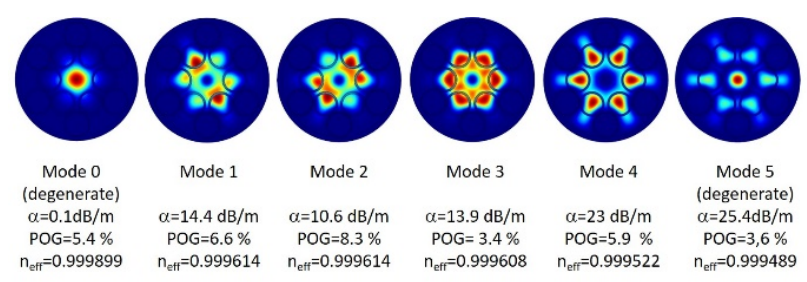

Fig. 4. Modes supported by the fiber structure at $\lambda=1.945 \mu \mathrm{m}(\alpha$ is the attenuation, POG is the fraction of optical power on the glass for each mode and $\mathrm{n}_{\text {eff }}$ is the effective index).

The first high order modes (HOMs) supported by the structure are cladding modes with leakage losses exceeding $10 \mathrm{~dB} / \mathrm{m}$, more than 2 order of magnitude greater than that of Mode 0 , while their fraction of POG is comparable. The only HOM with a spatial distribution mostly concentrated in the central air core is Mode 5 (which is a similar to a classical $\mathrm{LP}_{02}$ mode) but it has only a POG of $3.6 \%$ and over $25 \mathrm{~dB} / \mathrm{m}$ additional losses. Note also that all of these HOMs (including Mode 5) are very sensitive to fiber bending, unlike Mode 0 . This is why, for the purpose of this work, the optical fiber structure considered here can be considered "effectively single mode" after a propagation length of only few meters.

We now propose to evaluate the amplifying performances of the Tm-doped HC fiber described above. Numerical codes currently used for single-mode fiber are not adequate to the description of the non-conventional mode/dopant configuration of HCs. Thus, we have implemented a 3D spatially-resolved amplification numerical code, similar to the one developed in our group to model fewmode fiber amplifiers [26]. The mode intensity profiles at the pump and signal wavelengths, previously calculated using Comsol Multiphysics, are imported in a 2D $500 \times 500$ mesh, together with the transverse distribution of the $\mathrm{Tm}^{3+}$ ions. The addition of a 1D longitudinal meshing allows to solve the rate and propagation equations used to describe a two energy levels system (in-band pumping, i.e. pumping in the ${ }^{3} \mathrm{~F}_{4}$ first energy level and direct relaxation to the ${ }^{3} \mathrm{H}_{6}$ ground level). The equations can be solved in each element of the $500 \times 500$ mesh, in order to obtain the total transverse distributions of the population inversion and optical powers at different longitudinal positions of the fiber. In our analysis, we have considered that only the inner glass ring is Tmdoped (green circles in the inset of Fig. 5A). We have adopted the following parameters: Thulium concentration is fixed to $5 \times 10^{25}$ ions $/ \mathrm{m}^{3}$ in all the doped region, the lifetime of the upper energy level of $\mathrm{Tm}^{3+}$ ions is set to $430 \mu \mathrm{s}$, whereas emission and absorption cross-sections are extracted from the Optisystem software database [27]. Amplified Spontaneous Emission (ASE) has been considered in a $10 \mathrm{~nm}$ window around the signal wavelength. The confinement losses extracted from Fig. 2 $(0.112 \mathrm{~dB} / \mathrm{m}$ for the signal at $1945 \mathrm{~nm}$ and $0.139 \mathrm{~dB} / \mathrm{m}$ for the pump at $1565 \mathrm{~nm}$ ) have been considered. The results are shown in Fig. 5 and Fig. 6, where we have modelled a co-propagative configuration with $30 \mathrm{~W}$ input pump power and $0.5 \mathrm{~W}$ signal power.
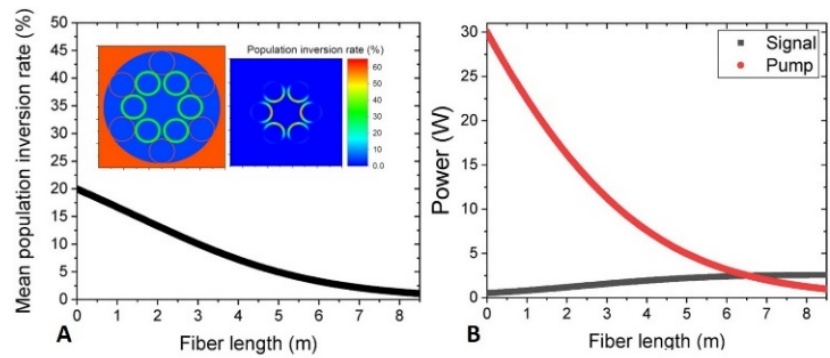

Fig. 5. A) Mean population inversion rate (averaging of the transverse distribution of the population inversion) as a function of the fiber length for the Tm-doped HC optical amplifier. Inset: it is shown the fiber geometry considered for the 3D spatially-resolved amplification (red stands for pure silica, green for Tm-doped silica and blue for air) and, on the right, is shown the transverse distribution of the population inversion rate at the fiber input. B) Evolution of signal $(\lambda=1945 \mathrm{~nm})$ and pump $(\lambda=1565 \mathrm{~nm})$ power along the fiber length. Input pump power has been fixed to $30 \mathrm{~W}$ and input signal power is set to $0.5 \mathrm{~W}$.

As can be seen in the inset of Fig. 5A population inversion can be realized locally in the Tm-doped zone at the fiber input and the average inversion rate is then about $20 \%$. An optimal fiber length of $8.5 \mathrm{~m}$ allows to obtain a gain of $7.1 \mathrm{~dB}$ for the signal wavelength (Fig. 5B and Fig. 6).

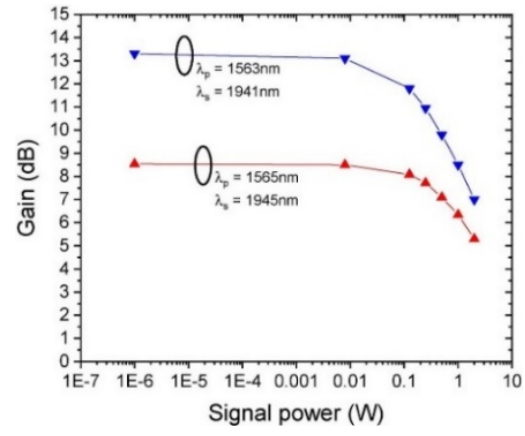

Fig. 6. Gain dependence of the Tm-doped HC amplifier on the signal power. Pump power has been fixed to $30 \mathrm{~W}$ for both red and blue plots. For the red line, signal and pump wavelengths are $1565 \mathrm{~nm}$ and $1945 \mathrm{~nm}$, respectively. For the blue line, they are $1563 \mathrm{~nm}$ and $1941 \mathrm{~nm}$.

The dependence of the optical gain on the signal power is presented in Fig. 6, for a different combination of wavelengths (giving rise to different overlaps). One can see that the results of Fig. 5 were obtained in the saturated signal regime and that small signal gain can reach $8.5 \mathrm{~dB}$ in the same conditions. However, 
when considering optical modes that present larger overlap with the Tm-doped region (i.e. modes at $1563 \mathrm{~nm}$ and $1941 \mathrm{~nm}$ for pump and signal - see Fig. 2), gain reaches $9.5 \mathrm{~dB}$ for $0.5 \mathrm{~W}$ signal power and $13 \mathrm{~dB}$ in small signal regime for a $6.5 \mathrm{~m}$-long HC Tmdoped fiber (all the other parameters being unchanged).

As we have demonstrated the possibility of realizing an HC optical fiber amplifier with the transversal structure of Fig. 1, it is interesting considering here an almost identical structure to be used as the passive optical fiber to directly interface with the Tm doped HC amplifier. Indeed, if the thickness $t_{1}$ of the internal ring of tubes of Fig. 1 is modified to $t_{1}^{*}=3.5 \mu \mathrm{m}$, then even the internal silica tubes would be in anti-resonance with respect to the signal wavelength. It is well known that the addition of extra antiresonant elements within the cladding area largely decreases fiber attenuation [28-31]. In this case the transmission of this ARF is shown in Fig. 7, where we can see that the leakage loss at 1.945 $\mu \mathrm{m}$ is about $0.1 \mathrm{~dB} / \mathrm{km}(1000$ times less than in the parent $\mathrm{HC}$ amplifier). Note that the presence of the sharp transmission peaks is related to the coupling between the fundamental-like mode and the silica cladding modes of the double cladding ring structure [13].

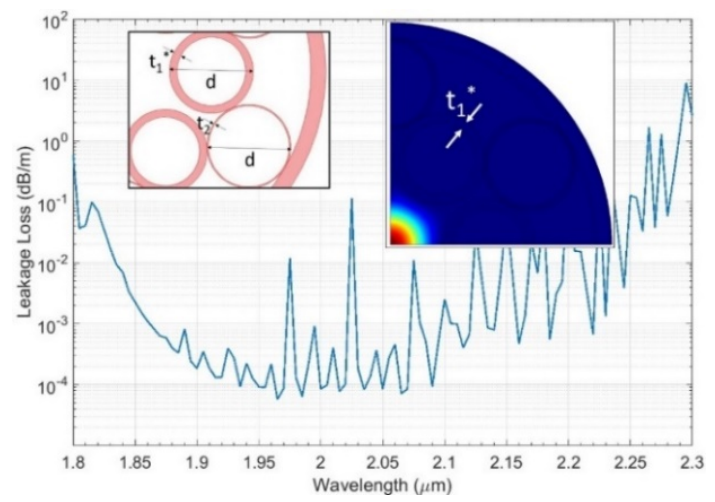

Fig. 7. Transmission spectrum of the fiber structure of Fig.1 with $t_{1}$ replaced by $t_{1}{ }^{*}=3.5 \mu \mathrm{m}$.

Ideally, this slight modification of the thickness of the internal silica tubes from $t_{1}$ to $t_{1}$ * could be realised by expanding (or reducing) the internal tube size adiabatically during or after fiber drawing, allowing an ideal mode matching between the active and the passive ARFs. The feasibility of structures with two antiresonant rings around a central air core has been demonstrated [31].

In conclusion, we have introduced a novel design for hollow core optical fibers which includes both resonant and anti-resonant cladding elements. We have numerically shown that this structure can have, at the same time, an increased overlap of the fundamental optical mode on the glass cladding region and a low optical attenuation. The combination of these two characteristics could be exploited for the realization of the first hollow core optical amplifier without the need of gas filling. We have evaluated the amplifying performances of effectively single mode Tmdoped HCs with good bend resistance and relatively short in length (less than $8.5 \mathrm{~m}$ ), which may provide small signal optical gains between $8.5 \mathrm{~dB}$ and $13 \mathrm{~dB}$. This amplifier may be connected with a HC based transmission link with minimal optical impedance, limiting mode mismatching and preserving low latency transmission.

We will also explore the use of our novel hollow core fiber structure in the fields of high power optical generation and mid-infrared laser sources.

Funding. This work is supported by the Excellence Research Chair Nathan from Région Hauts-de-France.

Acknowledgments. The authors acknowledge M. N. Zervas, S. Fevrier and 0 . Vanvincq for fruitful discussions.

\section{References}

1. W. Belardi, Fibers 7(5), 50 (2019).

2. I. A. Bufetov, A. F. Kosolapov, A. D. Pryamikov et al., Fibers 6(2), 39 (2018).

3. M. A. Duguay, et al., Appl. Phys. Lett. 49, 13-15 (1986).

4. J. L. Archambault, R. J. Black, S. Lacroix, and J. Bures, J. Lightwave Technol. 11, 416 (1993).

5. N. M. Litchinister, A. K. Abeeluck, C. Headley, and B. Eggleton, Opt. Lett. 27 (18), 1592-1594 (2002).

6. B. Debord, F. Amrani, L. Vincetti, F. Gérôme, F. Benabid, Fibers, 7 (2), 16 (2019).

7. W. Belardi and J. C. Knight, Opt. Express 22, 10091-10096 (2014).

8. X. Wang, D. Ge, W. Ding et al., Opt. Lett. 44, 2145-2148 (2019).

9. M. Michieletto, J. K. Lyngs $\varnothing$, C. Jakobsen et al., Opt. Express 24, 7103-7119 (2016).

10. A. L. S. Cruz, C. M. B. Cordeiro, M. A. R. Franco, Fibers, 6,43 (2018).

11. S.F. Gao, Y.Y. Wang, W. Ding, and P. Wang, Opt. Lett. 43, 13471350 (2018).

12. F. Wiegandt, P. N. Anderson, F. Yu et al., Optica 6, 442-447 (2019).

13. W. Belardi, J. C. Knight, Opt. Express 21, 21912-21917 (2013).

14. W. Belardi, J. C. Knight, OFC 2014, Th2A.45.

15. L. D. van Putten, E. N. Fokua, S. M. A. Mousavi et al., IEEE Photonics Technology Letters 29, 263-266 (2017).

16. M. R. Abu Hassan, F. Yu, Z. Wang, W. Belardi et al., CLEO 2015, SF1F.7.

17. S. Bateman, W. Belardi, F. Yu, C. Webb, W. J. Wadsworth, CLEO 2014 San Jose 8-13 June 2014.

18. A. V. V. Nampoothiri, A. M. Jones, C. Fourcade-Dutin et al., Opt. Mater. Express 2, 948-961 (2012).

19. W.Belardi, F. De Lucia, F. Poletti, P. J. Sazio, Opt. Letters, 42, 25352538 (2017).

20. A. Lewis, F. De Lucia, W. Belardi et al.,FiO 2018, paper FW6B.4.

21. J. Navarro-Arenas, I. Suarez, J. P. Martinez-Pastor et al., Nanomaterials 9(6), 868 (2019).

22. V. Bhagavatula, R. Bhat, G.E. Berkey et al., Proc. of SPIE Vol. 5335, 210-216 (2004).

23. J. Roberts, J. Broeng, A. Petersson, and K. P. Hansen, Proc. of SPIE Vol. 6102, 61020F (2006).

24. M. A Khamis, K. Ennser, IEEE J. Light, Techn. 34, 5675-5681 (2016).

25. P. J. Roberts, F. Couny, H. Sabert et al., Opt. Express 13, 236-244 (2005).

26. G. Le Cocq, L. Bigot, A. Le Rouge, M. Bigot-Astruc, P. Sillard, C. Koebele, M. Salsi, et Y. Quiquempois, Optics Express 20 24, p. 27051-27061 (2012).

27. https://optiwave.com/optisystem-overview/

28. W. Belardi and J. C. Knight, Opt. Letters 39, 1853-1856 (2014).

29. W. Belardi and J. C. Knight, OFC 2014, paper Th2A.45 (Supplementary Material).

30. S. F. Gao, Y. Y. Wang, W. Ding et al., Nat. Commun. 9, 2828 (2018).

31. X. Huang, W. Qi, D. Ho et al., Opt. Express 24, 7670-7678 (2016). 


\section{References}

1. W. Belardi, "Hollow-core optical fibers," Fibers 7(5), 50 (2019).

2. I. A. Bufetov, A. F. Kosolapov, A. D. Pryamikov, A. V. Gladyshev, A. N. Kolyadin, A. A. Krylov, Y. P. Yatsenko, A. S. Biriukov, "Revolver Hollow Core Optical Fibers," Fibers 6(2), 39 (2018).

3. M. A. Duguay, et al., "Antiresonant reflecting optical waveguides in $\mathrm{SiO}_{2} \mathrm{Si}$ multilayer structures," Appl. Phys. Lett. 49, 13-15 (1986).

4. J. L. Archambault, R. J. Black, S. Lacroix, and J. Bures, "Loss calculations for antiresonant waveguides," J. Lightwave Technol. 11, 416 (1993).

5. N. M. Litchinister, A. K. Abeeluck, C. Headley, and B. Eggleton, "Antiresonant reflecting photonic crystal optical waveguides," Opt. Lett. 27(18),1592-1594 (2002).

6. B. Debord, F. Amrani, L. Vincetti, F. Gérôme, F. Benabid, "Hollow-Core Fiber Technology: The Rising of "Gas Photonics". Fibers, 7 (2), 16 (2019).

7. W. Belardi and J. C. Knight, "Hollow antiresonant fibers with low bending loss", Opt. Express 22, 10091-10096 (2014).

8. Xiaocong Wang, Dawei Ge, Wei Ding, Yingying Wang, Shoufei Gao, Xin Zhang, Yizhi Sun, Juhao Li, Zhangyuan Chen, and Pu Wang, "Hollow-core conjoined-tube fiber for penalty-free data transmission under offset launch conditions," Opt. Lett. 44, 2145-2148 (2019).

9. M. Michieletto, J. K. Lyngs $\varnothing$, C. Jakobsen, J. Lægsgaard, O. Bang, and T. T. Alkeskjold, "Hollow-core fibers for high power pulse delivery," Opt. Express 24, 7103-7119 (2016).

10. A. L. S. Cruz, C. M. B. Cordeiro, M. A. R. Franco, "3D Printed Hollow-Core Terahertz Fibers," Fibers, 6, 43 (2018).

11. S.F. Gao, Y.Y. Wang, W. Ding, and P. Wang, "Hollow-core negative-curvature fiber for UV guidance," Opt. Lett. 43, 1347-1350 (2018).

12. Florian Wiegandt, Patrick N. Anderson, Fei Yu, Daniel J. Treacher, David T. Lloyd, Peter J. Mosley, Simon M. Hooker, and Ian A. Walmsley, "Quasi-phase-matched high-harmonic generation in gas-filled hollow-core photonic crystal fiber," Optica 6, 442-447 (2019).

13. W. Belardi, J. C. Knight, "Effect of core boundary curvature on the confinement losses of hollow antiresonant fibers", Opt. Express 21, 21912-21917 (2013).

14. W. Belardi, J. C. Knight, "Negative curvature fibers with reduced leakage loss," OFC 2014, Th2A.45.

15. L. D. van Putten, E. N. Fokua, S. M. A. Mousavi, W. Belardi, S. Chaudhuri, J. V. Badding, F. Poletti, "Exploring the Effect of the Core Boundary Curvature in Hollow Antiresonant Fibers," IEEE Photonics Technology Letters 29, 263-266 (2017).

16. M. R. Abu Hassan, F. Yu, Z. Wang, W. Belardi, W. J. Wadsworth, J. C. Knight, Synchronously pumped mid-IR hollow core fiber gas laser. CLEO 2015, SF1F.7.

17. S. Bateman, W. Belardi, F. Yu, C. Webb, W. J. Wadsworth, CLEO 2014 San Jose 8-13 June 2014.
18. A. V. Vasudevan Nampoothiri, Andrew M. Jones, C. Fourcade-Dutin, Chenchen Mao, Neda Dadashzadeh, Bastian Baumgart, Y.Y. Wang, M. Alharbi, T. Bradley, Neil Campbell, F. Benabid, Brian R. Washburn, Kristan L. Corwin, and Wolfgang Rudolph, "Hollow-core Optical Fiber Gas Lasers (HOFGLAS): a review [Invited]," Opt. Mater. Express 2, 948-961 (2012).

19. W.Belardi, F. De Lucia, F. Poletti, P. J. Sazio,"Composite material hollow antiresonant fibers," Opt. Letters, 42, 25352538 (2017).

20. A. Lewis, F. De Lucia, W. Belardi ,C. Huang, J. R. Hayes, F. Poletti, D. Hewak, P.J. Sazio," Composite material hollow core optical fiber electro-modulation,"FiO 2018, paper FW6B.4.

21. J . Navarro-Arenas, I. Suarez, J. P. Martinez-Pastor, A. Ferrando, A. F Gualdrón-Reyes, I. Mora-Seró, S. Gao, Y. Wang, P.Wang, Z. Sun," Optical Amplification in HollowCore Negative-Curvature Fibers Doped with Perovskite $\mathrm{CsPbBr}_{3}$ Nanocrystals," Nanomaterials 9(6), 868 (2019).

22. V. Bhagavatula, R. Bhat, G.E. Berkey, X. Chen, M.J. Dejneka, M. Gallagher, L.C. Hughes, K.W. Koch, J. Koh, M.J. Li, X. Liu, D.A. Nolan, M.H. Rasmussen, C.L. Tennent, N. Venkataraman, D.T. Walton, J. Wang, C.E. Zah, L.A. Zenteno "Progress in High Power Fiber Lasers," Proc. of SPIE Vol. 5335, 210-216 (2004).

23. J. Roberts, J. Broeng, A. Petersson, and K. P. Hansen,"Amplification in hollow core photonic crystal fibers," Proc. of SPIE Vol. 6102, 61020F (2006).

24. M. A. Khamis and K. Ennser, "Theoretical Model of a Thulium-Doped Fiber Amplifier Pumped at $1570 \mathrm{~nm}$ and 793 $\mathrm{nm}$ in the Presence of Cross Relaxation," J. Lightwave Technol. 34, 5675-5681 (2016).

25. P. J. Roberts, F. Couny, H. Sabert, B. J. Mangan, D. P. Williams, L. Farr, M. W. Mason, A. Tomlinson, T. A. Birks, J. C. Knight and P. St. J. Russell, "Ultimate low loss of hollow-core photonic crystal fibres," Opt. Express 13, 236-244 (2005).

26. G. Le Cocq, L. Bigot, A. Le Rouge, M. Bigot-Astruc, P. Sillard, C. Koebele, M. Salsi, et Y. Quiquempois, "Modeling and characterization of a few-mode EDFA supporting four mode groups for mode division multiplexing," Optics Express 20 24, p. 27051-27061 (2012)

27. https://optiwave.com/optisystem-overview/

28. W. Belardi and J. C. Knight, "Hollow antiresonant fibers with reduced attenuation", Opt. Letters 39, 1853-1856 (2014).

29. W. Belardi and J. C. Knight, "Negative curvature fibers with reduced leakage loss", OFC 2014, paper Th2A.45 (Supplementary Material).

30. S. F. Gao, Y. Y. Wang, W. Ding, D. L. Jiang, S. Gu, X. Zhang, and P. Wang, Nat. Commun. 9, 2828 (2018).

31. X. Huang, W. Qi, D. Ho, K. Yong, F. Luan, and S. Yoo, "Hollow core anti-resonant fiber with split cladding," Opt. Express 24, 7670-7678 (2016). 